\title{
Association of bacteria and viruses with wheezy episodes in young children: prospective birth cohort study
}

We have just been notified by the authors of this 2010 paper, Hans Bisgaard and colleagues (BMJ 2010;341:c4978, doi:10. 1136/bmj.c4978), that the Funding statement should have included the following declaration: "This work was funded in part by the MoRIAE European Research Council FP7 Advanced Investigator award to SLJ, grant number 233015."

Cite this as: BMJ 2011;343:d8113

๑ BMJ Publishing Group Ltd 2011 